\title{
Inclusive jet cross-sections and dijet azimuthal decorrelations with DØ
}

\author{
Raimund Ströhmer*† \\ LMU Muenchen, Germany \\ E-mail: Raimund.Stroehmer@Physik. Uni-Muenchen.de
}

We present a preliminary measurement of the inclusive jet cross-sections based on an integrated luminosity of $378 \mathrm{pb}^{-1}$ acquired with the DØ detector between 2002 and 2004 at a center of mass energy of $\sqrt{s}=1.96 \mathrm{TeV}$ and a measurement of azimuthal dijet decorrelations based on an integrated luminosity of $150 \mathrm{pb}^{-1}$. The cross section measurements are based on an iterative cone algorithm with a cone size of $R=0.7$. They are performed in two rapidity bins between 0.0 and 0.8. The measurements are in good agreement with next to leading order calculations.

The azimuthal angle between the two leading jets is sensitive to higher order QCD effects. The measurement of dijet azimuthal decorrelations therefore probes these effects without explicitly reconstructing more than two jets. Except for large azimuthal angles where soft effects are important the measurements are well described by the next to leading order perturbation theory.

International Europhysics Conference on High Energy Physics

July 21st - 27th 2005

Lisboa, Portugal

\footnotetext{
*Speaker.

${ }^{\dagger}$ For the DØ Collaboration.
} 


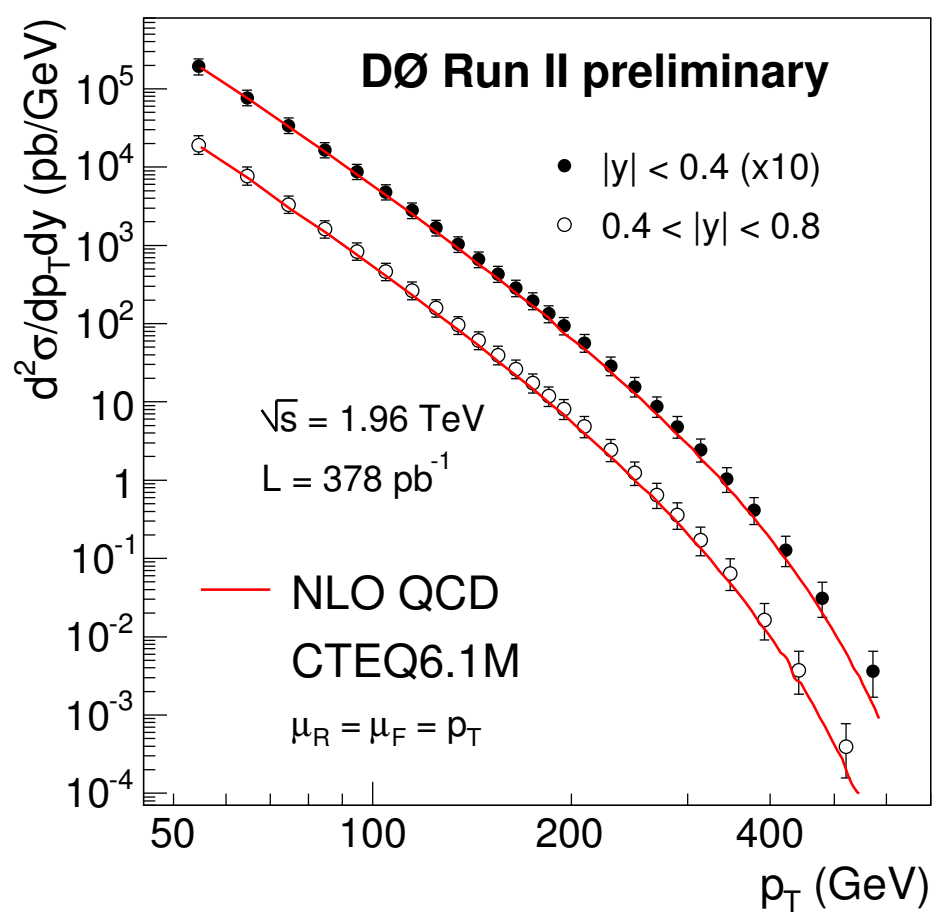

Figure 1: The inclusive jet cross section in two regions of jet rapidity. The error bars indicate the total experimental error. The predictions from NLO pQCD are overlaid on the data.

\section{Inclusive jet cross-sections}

Both the increased center of mass energy and the higher integrated luminosity made it possible to measure the inclusive jet cross section at higher transverse jet momenta as compared to Run I. At $p_{T}=500 \mathrm{GeV}$ for example the cross section is according to $\mathrm{pQCD}$ calculations expected to increase by $300 \%$ due to the change of center of mass energy from $\sqrt{s}=1.8 \mathrm{TeV}$ in Run I to $\sqrt{s}=1.96 \mathrm{TeV}$ in Run II. At the highest jet $p_{T}$ quark anti-quark fusion diagrams dominate but at $p_{T}=500 \mathrm{GeV}$ the quark-gluon diagrams still contribute with about $30 \%$ to the cross section and therfore give sensitivity to the gluon density in the proton.

The jets are reconstructed with an iterative cone algorithm [1] with a cone size of $R=0.7$. The inclusion of the midpoints between jets as additional seeds avoids infrared instabilities of simpler cone algorithms.

The dominant systematic uncertainty is due to the understanding of the jet energy calibration. Due to the steeply falling cross section spectrum shifts in the energy scale cause large effects on the measured cross section. The jet energy scale is estimated from the $p_{T}$ inbalance in photon plus jet events [2]. The effects of the underlying event and hadronization are estimated to be small. Therefore the measured cross sections are only corrected for the jet energy measurement selection, for efficiencies and for bin migrations due to the $p_{T}$ resolution. Figure 1 shows the measurements in two rapidity bins $(|y|<0.4$ and $0.4<|y|<0.8)$. The measurements are compared with a NLO QCD 

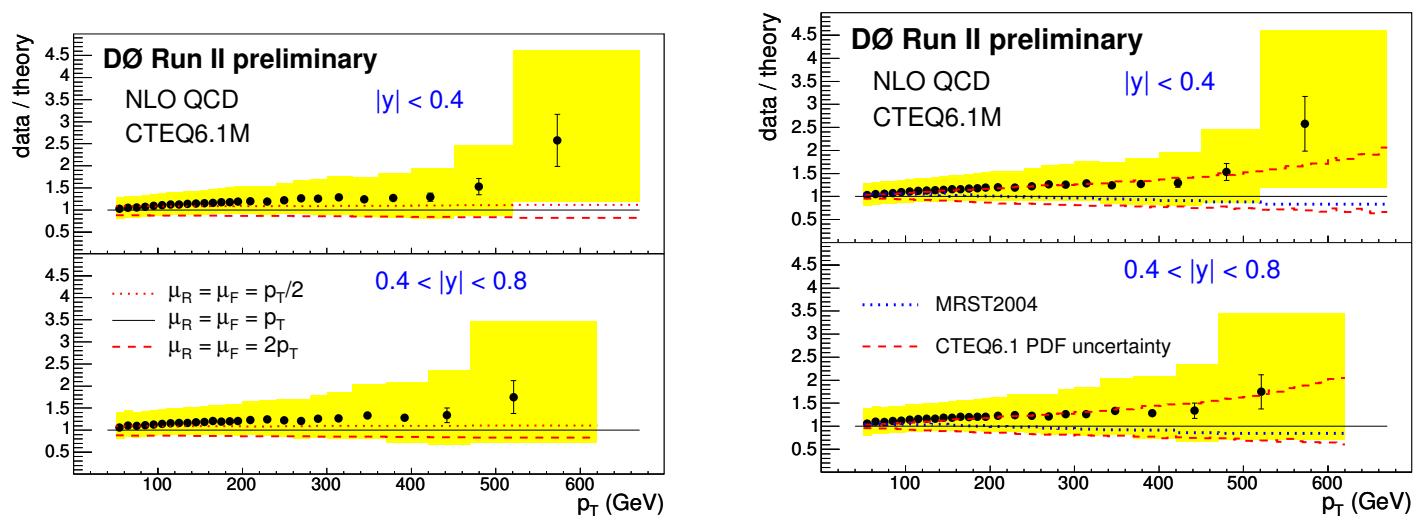

Figure 2: Ratio of inclusive jet cross section to the predictions from NLO pQCD. The bands indicate the total experimental error. The dashed and dotted lines indicate uncertainties due to the renormalisation and factorization scale and uncertainties due to the PDF's.

calculation using the program NLOJET++ [3] and PDFs from CTEQ6.1M [4] and MRST2004 [5]. In Figure 2 the ratio of the measured and the predicted cross section are shown. The band indicates the total experimental uncertainty while the dashed and dotted lines indicate uncertainties due to the renormalisation and factorization scale and uncertainties due to PDF uncertainties.

\section{Azimuthal decorrelations}

The azimuthal angle between the two highest $p_{T}$ jets can be used to study higher order effects without explicitly reconstructing additional jets. The distribution of the azimuthal angle close to $180^{\circ}$ is strongly effected by soft QCD effects. On the other hand large deviations from the back to back topology are caused by hard parton emissions. Angles between the two highest $p_{T}$ jets smaller than $120^{\circ}$ can only be achieved if more than one hard parton is emitted.

The observable was defined as the differential cross section in $\Delta \phi$ normalized to the cross section integrated over the full $\Delta \phi$ range. This normalization reduces both theoretical and experimental uncertainty especially the uncertainty due to the energy scale. As in the cross section measurement jets are reconstructed with an iterative cone algorithm [1] with a cone size of $R=0.7$. The two highest $p_{T}$ jets are required to have rapidities $|y|<0.5$. The second highest $p_{T}$ jet was required to have $p_{T}>40 \mathrm{GeV}$. The angular distribution is then measured in bins of the $p_{T}$ of the highest $p_{T}$ jet. The measurement is compared in Figure 3 with LO and NLO pQCD calculations [3, 4] and with the Monte Carlo generators HERWIG [6] and PYTHIA [7]. For angles close to $180^{\circ}$ the pQCD calculations become numerically unstable and are not shown. In this region soft QCD effects are large. Except for that region the NLO prediction describes the measurements well. While the leading order prediction can neither describe the region of small angles (where more than one parton is emitted) nor the region of large angles. HERWIG (version 6.505) also describes the data well while PYTHIA with the default parameters of version 6.225 does not describe the data well. The agreement can however be greatly improved if the initial state radiation is increased (the parameter PARP(67) was increased form 1.0 to 4.0). 

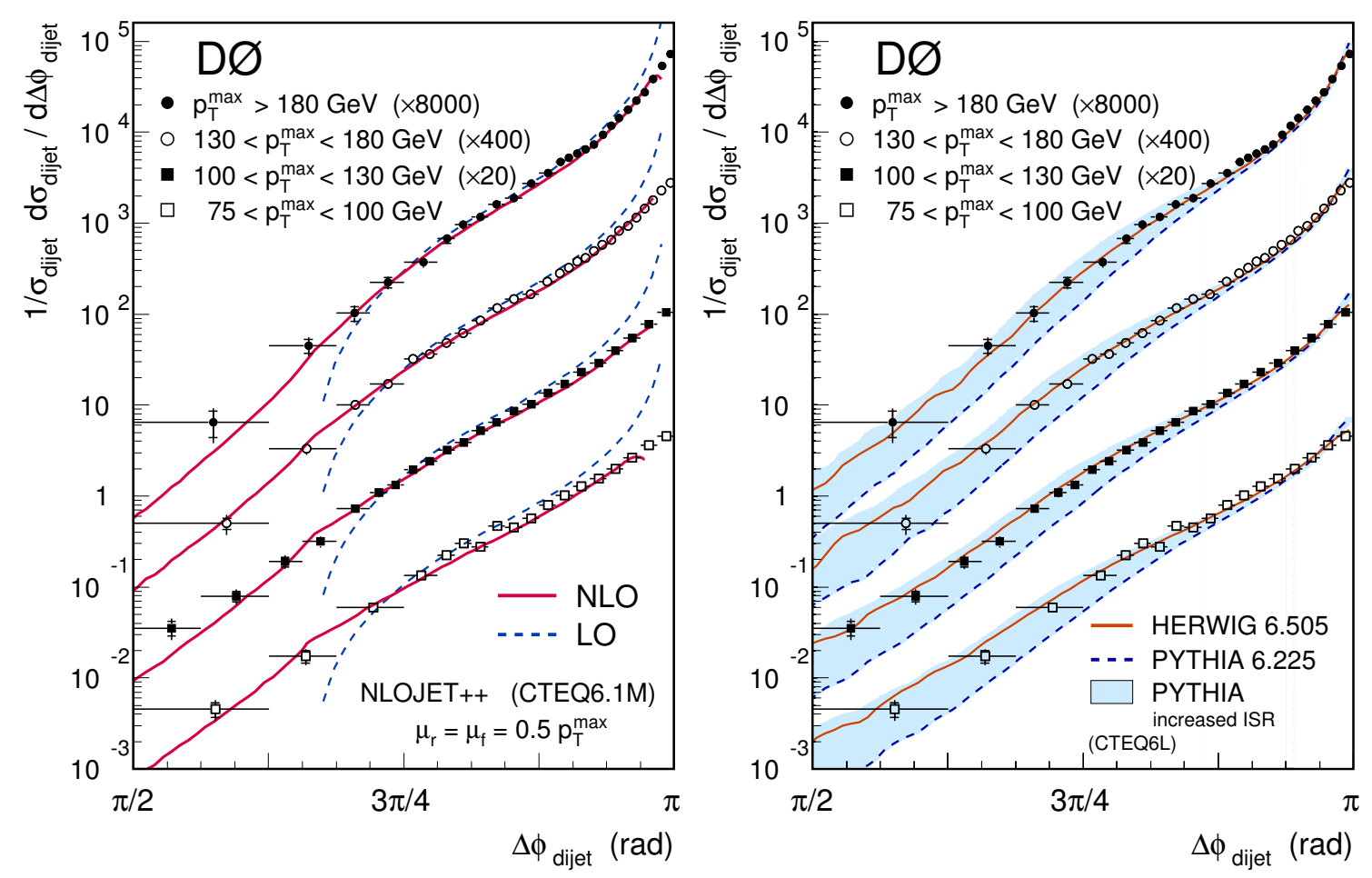

Figure 3: The $\Delta \phi_{\text {dijet }}$ distribution in four regions of $p_{T}^{\max }$ compared to NLO an LO pQCD predictions and to the predictions of QCD shower Monte Carlos.

\section{References}

[1] G.C. Blazey et. al., in Proceedings of the Workshop: "QCD and Weak Boson Physics in Run II", edited by U. Baur, R. K. Ellis, and D. Zeppenfeld, Batavia, Iiinois (2000) p. 47. See Section 3.5 for details.

[2] B. Abbott et. al. (DØ Collaboration), Nucl. Inst. and Meth, A 424, 352 (1999).

[3] Z. Nagy, Phys. Rev. Lett. 88, 122003 (2002); Z. Nagy, Phys. Rev. D 68, 094002 (2003).

[4] K. Pumplin et. al., JHEP 0207, 12 (2002); D. Stump et. al. JHEP 0310, 046 (2003)

[5] A.D. Martin et. al. Phys. Lett. B 604, 61 (2004).

[6] G. Marchesini et. al. Comp. Phys. Comm. 67,465 (1992); G. Corecella et. al. JHEP 0101, 010 (2001).

[7] T. Sjöstrand et. al. Comp. Phys. Comm. 135,238 (2001); 